\title{
Validation of 3D Model-Based Maximum-Likelihood Estimation of Normalisation Factors for Partial Ring Positron Emission Tomography
}

\author{
Tahereh Niknejad, Stefaan Tavernier, Joao Varela and Kris Thielemans, Senior Member, IEEE
}

\begin{abstract}
The next generation of organ specific Positron Emission Tomography (PET) scanners, e.g. for breast imaging, will use partial ring geometries. We propose a component-based Maximum-Likelihood (ML) estimation of normalisation factors for 3D PET data reconstruction applicable to partial ring geometries. This method is based on the Software for Tomographic Image Reconstruction (STIR) for full ring PET and is validated for a stationary partial ring scanner. The model includes the estimation for crystal efficiencies and geometric factors. The algorithm is validated using Maximum Likelihood Estimation Method (MLEM) based 3D reconstruction in STIR using Geant4 Application for Tomographic Emission (GATE) simulation data for full and partial ring scanners and experimental data from a demonstrator with partial ring geometry. The uniformity of the reconstructed images of simulated cylindrical and NEMAIQ phantoms in both scanner geometries and the image of a line source in the partial ring demonstrator is assessed. The results have shown that uniform images in both axial and transaxial directions are obtained after applying the estimated normalisation factors. The accuracy of the algorithm is validated by comparing the normalisation factors between the full and partial ring systems in simulation. We have shown that the estimated normalisation factors are almost identical, even though the separate components are not. This proves that the ML estimation of the 3D normalisation factors is valid and can be applied to the partial ring scanner.
\end{abstract}

\section{INTRODUCTION}

$\mathbf{P}$ ET data reconstruction requires an accurate estimation of the normalisation factors to take the detection sensitivity of different lines of responses (LOR) into account. Normalisation factors are multiplicative parameters to the measured data. The motivation for this project was to develop a 3D image reconstruction software for our recent developed PET demonstrator with partial ring geometry which is a test machine for the next generation of organ specific PET scanners, e.g. for breast imaging with partial ring design [1]. This machine is

This collaboration is supported by the European project COST Action TD1401 Fast Advanced Scintillator Timing (FAST) and the EPSRC (EP/M022587/1) Collaborative Computational Project in Positron Emission Tomography and Magnetic Resonance imaging (CCP-PET-MR) (www.ccppetmr.ac.uk). The work is carried out at Institute of Nuclear Medicine, UCL hospital, London, UK.

T. Niknejad (e-mail: tniknejad@lip.pt) is with the Laboratory of Instrumentation and Experimental Particle Physics (LIP), Lisbon, Portugal.

S. Tavernier is with Vrije Universiteit Brussel, Belgium and PETsys Electronics, Lisbon, Portugal.

J. Varela is with PETsys Electronics, Lisbon, Portugal.

K. Thielemans is with Institute of Nuclear Medicine, University College London, UK. stationary, so the angular coverage is not complete. Previously, a component based method to estimate normalisation factors based on maximising the Poisson log-likelihood for 2D PET data reconstruction has been developed [2]. Based on that algorithm, a 3D normalisation factor estimation is derived in this work for full ring PET and validated for stationary partial ring scanner. The reconstruction is done in open source Software for Tomographic Image Reconstruction STIR [4]. The normalisation factors are split into two components including the crystal efficiencies and geometric effects to reduce the number of components and the noise on their estimation. The components are geometric $G_{i j}$ (representing the change in sensitivity due to the radial position of the detector pair and relative position of each detector pair within a block) and crystal efficiency $\epsilon_{i} \epsilon_{j}$ factors. To reduce the number of independent geometric factors, we imposed symmetries according to the scanner geometry of a full ring system.

\section{THEORY}

The measured PET data have Poisson distribution. The data are modelled as

$$
M_{i j}=\epsilon_{i} \epsilon_{j} G_{i j} A_{i j}
$$

where $M_{i j}$ are the mean measured number of coincidences measured between crystal $i$ and $j$ in a scanner ring. These are equal to the product of two normalisation components (crystal efficiency factors $\epsilon_{i} \epsilon_{j}$ and geometric factor $G_{i j}$ ) and the ideal measurements model $A_{i j}$ (source distribution). A likelihood function $\mathrm{L}$ is defined, which is the probability that count $m_{i j}$ (measured data) are observed, given a known object and the normalisation factors.

$$
\log (L)=\sum_{i j}\left(m_{i j} \log \left(M_{i j}\right)-M_{i j}-\log \left(m_{i j}\right) !\right)
$$

In this method the measured object $\left(A_{i j}\right)$ is known and we want to estimate the normalisation factors. This is done by maximising $\mathrm{L}$ with respect to the normalisation factors. For efficiency factor, the maximum likelihood (ML) is reached when 


$$
\epsilon_{k}=\frac{\sum_{j} m_{k j}}{\sum_{j} \epsilon_{j} G_{k j} A_{k j}}
$$

which means the sum of coincidence counts seen by one detector is proportional to its efficiency. For geometric factors, ML is reached when

$$
G_{k l}=\frac{\sum_{i j} m_{i j}}{\sum_{i j} \epsilon_{i} \epsilon_{j} A_{i j}}
$$

where the sum is over all $\mathrm{ij}$ detector pairs that have the same geometric factor as $G_{k l}$. To find the equivalent LORs, we implemented mirror, rotational and translational symmetries in $3 \mathrm{D}$ for a full ring scanner in the algorithm (figure 1). For the partial ring system, we still keep the same symmetries and have virtual crystals (with zero detection efficiency) in the missing part of the detector ring. For the definition of the virtual crystal please refer to section $3 . G_{k l}$ is only estimated in one reference module for half the crystals in the module in transaxial direction and all the crystals in axial directions to cover all the oblique LORs. The calculated geometric factors for the selected LORs are then applied to the equivalent LORs according to the symmetries. An iterative Coordinate Ascent ML algorithm is used to solve these equations where one of the $\epsilon_{i}$ or $G_{i j}$ is updated given estimates to all the normalisation factors. For more details on the algorithm see the references [2][3]. The estimated normalisation factors are then included in the sensitivity image. For each voxel, the sensitivity image represents the total probability of detection of an event originating in that voxel. Without having the normalisation factors, it is computed by backprojecting projection data with all elements set to 1 . Having the normalisation factors, the sensitivity image is the geometric backprojection of the normalisation factors.

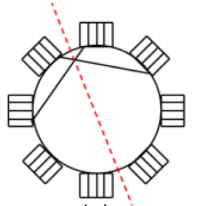

(a)

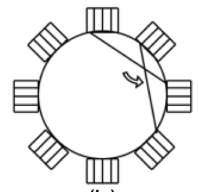

(b)

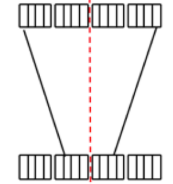

(c)

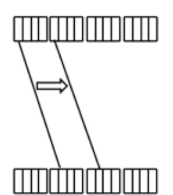

(d)
Fig. 1. Schematic view of the mirror and rotational symmetries in transaxial plane in (a) and (b) respectively and mirror and translational symmetries in axial planes in (c) and (d) respectively.

\section{Simulation AND EXPERIMENTAL STUDIES}

Using the method described above, we estimated the normalisation factors for full and partial ring scanners. Data were acquired with the demonstrator with partial ring geometry and in simulation with Geant4 Application for Tomographic Emission (GATE) [5]. Two scanners, full ring and partial ring geometry, were simulated. The simulated partial ring scanner matches the real scanner.

\section{A. Scanner design}

The PET demonstrator (figure 2a) ring has an inner diameter of $235 \mathrm{~mm}$ (crystal to crystal distance) and can accommodate 24 detector modules (figure 2b). However, the demonstrator is only equipped with 16 detector modules forming two arcs of 8 modules facing each other. Each detector module has 4 by 2 matrices of $4 \times 4$ LYSO scintillating crystals (figure $2 \mathrm{c}$ ), each measuring $3.1 \times 3.1 \times 15 \mathrm{~mm}^{3}$. The LYSO crystal array has a pitch of $3.2 \mathrm{~mm}$. The gaps between two adjacent matrices is about the same size of the crystal pixel. The demonstrator is also simulated in GATE with the current partial ring geometry (figure 3a) and with the full ring geometry having all the 24 modules (figure 3b). The efficiencies of all the crystals is almost identical in the simulation. In particular, there is only a small variation within each module as optical photon transport is not included in the simulation.

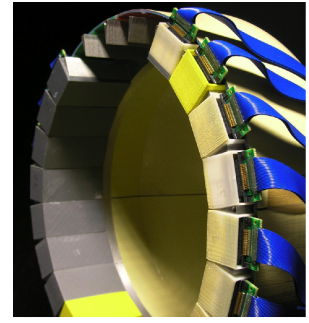

(a)

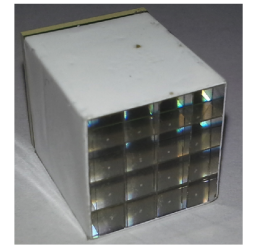

(c)

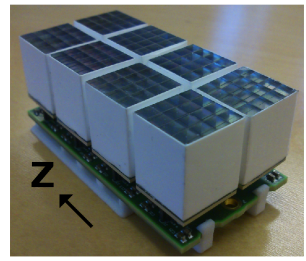

(b)

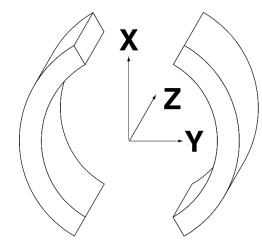

(d)
Fig. 2. Pictures of (a) the demonstrator, (b) a detector module and (c) a crystal matrix. The axes orientation is shown in (d).

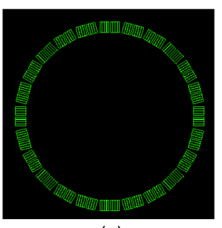

(a)

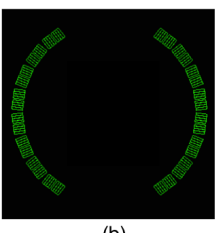

(b)
Fig. 3. Schematic transaxial picture of the simulated (a) full ring and (b) partial ring scanners.

In STIR, the scanner geometry is defined in the library to create a virtual scanner around the object. The acquired data will be then assigned to these virtual crystals to be reconstructed. To add the gaps between the matrices, in the scanner definition we assumed we have 5 by 5 crystals per module, which implies 4 by 4 physical crystals and a gap at the 5th crystal in both axial and transaxial directions where the counts are zero. These gaps are removed in applying the symmetries for geometric factors estimation. The scanner definition for the partial ring is the same as the full ring with zero detection efficiency for the virtual crystals in the missing parts of the detector ring. 


\section{B. Sources and phantoms}

A rotating line source in the demonstrator and a thin ring source in the simulation with the same length as the scanner and $110 \mathrm{~mm}$ radius were used for estimating the normalisation factors. A cylindrical phantom with $50 \mathrm{~mm}$ radius and $60 \mathrm{~mm}$ length with uniform activity and a NEMA Image Quality (IQ) phantoms [6] were used for simulation studies to evaluate the effect of the normalisation on the image uniformity. Radioactive sources were simulated as back-to-back gamma rays. A ${ }^{68} \mathrm{Ge}$ line source with nearly the same length as the scanner was used to study the uniformity of the image with the demonstrator.

\section{RESUlts}

\section{A. Simulation}

1) Validation of algorithm: To validate the algorithm in $3 \mathrm{D}$, the product of the normalisation components (efficiency and geometric factors) for the full ring and the partial ring were compared, by plotting a profile through one of the normalisation sinograms for both scanners. Data were obtained with simulation. The product of the estimated normalisation factors are almost identical even though the separate components are not. figure 4 and figure 5 show the efficiency and geometric profiles respectively while figure 6 is the product of these two factors for selected bins. This proves that the ML estimation of the 3D normalisation factors is valid and can be applied to the partial ring scanner.

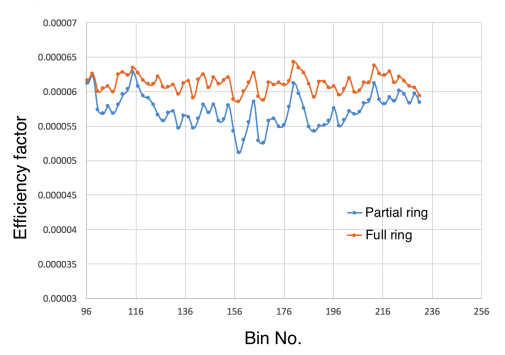

Fig. 4. Efficiency factor for selected bins for partial ring and full ring scanners.

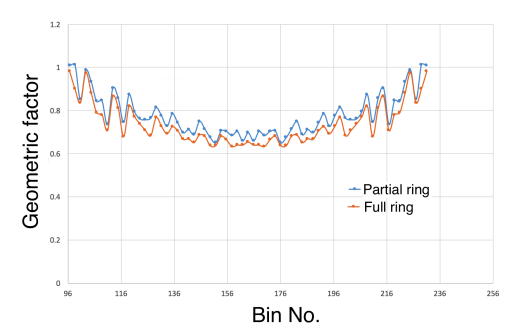

Fig. 5. Geometric factor for selected bins for partial ring and full ring scanners.

\section{B. Sensitivity image}

The sensitivity image before and after applying the normalisation factors are seen in figure 7. Since the scanner definition is STIR for full and partial ring scanners is the same and

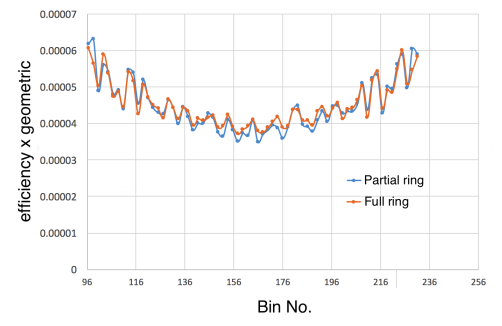

Fig. 6. Multiplication of the normalisation factors for selected bins for partial ring and full ring scanners.

is a full ring, without applying the estimated normalisation factors the sensitivity image for both scanners is computed by backprojecting projection data with all elements set to 1 (also for the gaps). Therefore, without normalisation factors estimation, one sensitivity image as shown in figure $7 \mathrm{a}$ is considered for full and partial ring scanners. After applying the estimated normalisation factors, the sensitivity images for full ring and partial ring scanners are the geometric backprojection of the normalisation factors as seen in figure $7 \mathrm{~b}$ and figure $7 \mathrm{c}$ respectively. The effect of the gaps can also be seen clearly in the sensitivity images.

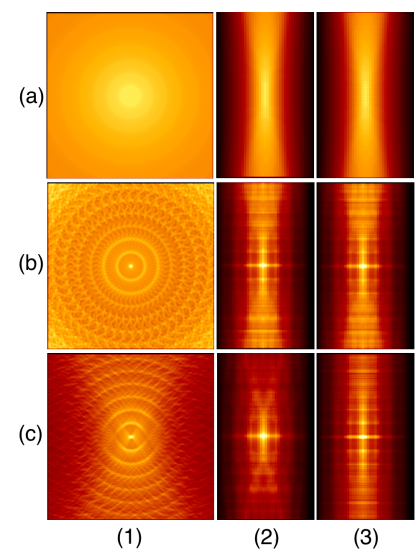

Fig. 7. Sensitivity images for (1) full and partial ring scanners before applying normalisation factors and (2) and (3) after applying normalisation factors for full ring and partial ring scanners respectively. (a), (b) and (c) are for xy, yz and $x z$ planes respectively.

\section{Image uniformity}

The reconstructed images of a uniform cylinder and NEMA IQ phantom with a full ring scanner and partial ring scanner are presented. Images are reconstructed with ML-EM algorithm with 120 iterations. Figure 8 and figure 9 show the reconstructed images of the uniform cylinder with and without normalisation factors and their respective profiles along the red lines for the full ring scanner in transaxial and axial planes respectively. Similarly, the reconstructed images of a uniform cylinder is shown for a partial ring scanner in figure 10 and figure 11 for transaxial and axial planes respectively.

Moreover, uniform images of NEMA IQ phantom were achieved with full and partial ring scanners after applying the normalisation factors as shown in figure 12 and figure 13 respectively. 


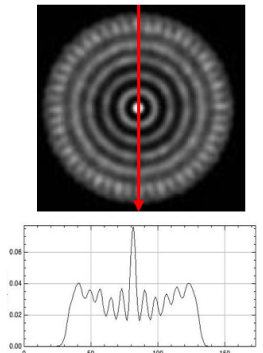

(a)
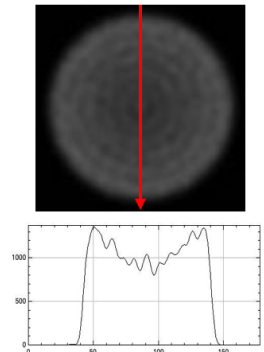

(b)

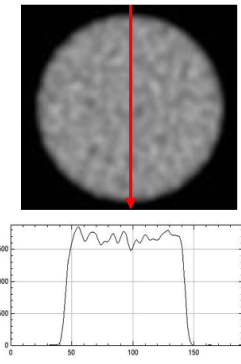

(c)
Fig. 8. Reconstructed images of a uniform cylinder in the full ring scanner and the corresponding profiles along the red arrows in xy plane (a) without normalisation factors, (b) with efficiency factors and (c) with both efficiency and geometric factors.

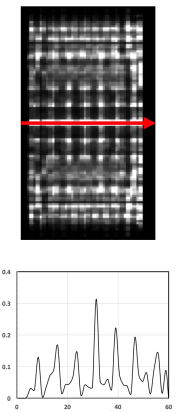

(a)
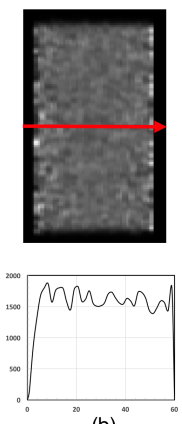

(b)
Fig. 9. Reconstructed images of a uniform cylinder in the full ring scanner and the corresponding profiles along the red arrows in $\mathrm{xz}$ plane (a) without normalisation factors and (b) with normalisation factors.
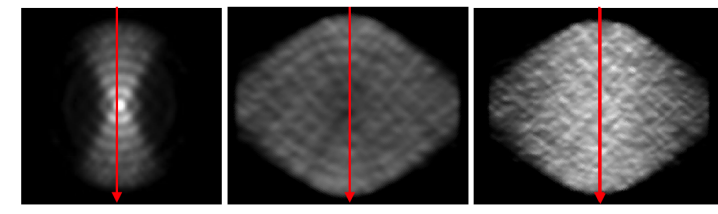

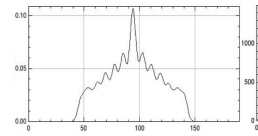

(a)

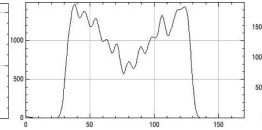

(b)

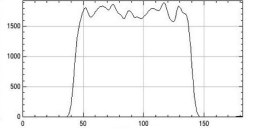

(c)
Fig. 10. Reconstructed images of a uniform cylinder in the partial ring scanner and the corresponding profiles along the red arrows in xy plane (a) without normalisation factors, (b) with efficiency factors and (c) and with both efficiency and geometric factors.

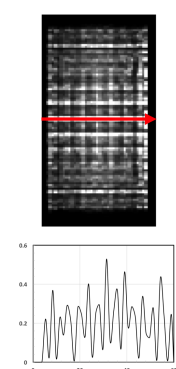

(a)
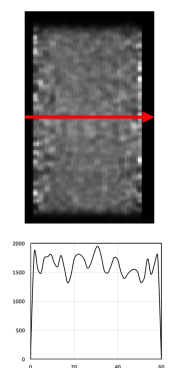

(b)
Fig. 11. Reconstructed image of a uniform cylinder in the partial ring scanner and the corresponding profile along the red arrows in $\mathrm{xz}$ plane (a) without normalisation factors and (b) with normalisation factors (b).

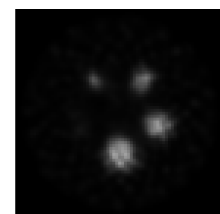

(a)

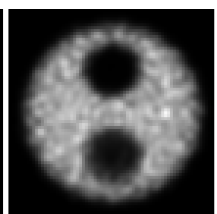

(b)

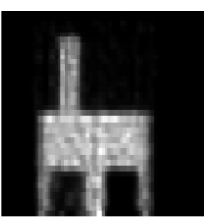

(c)
Fig. 12. Reconstructed image of the NEMA QI phantom in the full ring scanner (a) and (b) in xy and (c) xz planes with normalisation factors. Data is corrected for attenuation, randoms and scatters.

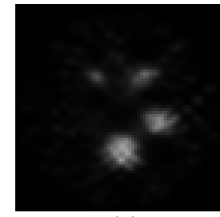

(a)

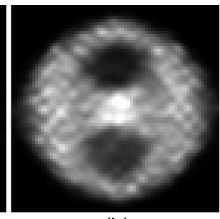

(b)

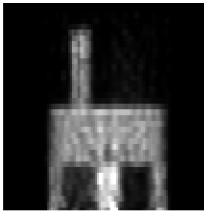

(c)
Fig. 13. Reconstructed image of the NEMA QI phantom in the partial ring scanner (a) and (b) in xy and (c) xz planes with normalisation factors. Data is corrected for attenuation, randoms and scatters.

\section{Experimental}

A $1.5 \mathrm{~mm}$ diameter and $60 \mathrm{~mm}$ long ${ }^{68} \mathrm{Ge}$ line source placed in the centre of the demonstrator along the axis of the scanner and its image is reconstructed after aplying the estimated normalisation factors as shown in figure 14. The longitudinal profile shows the uniformity of the image.
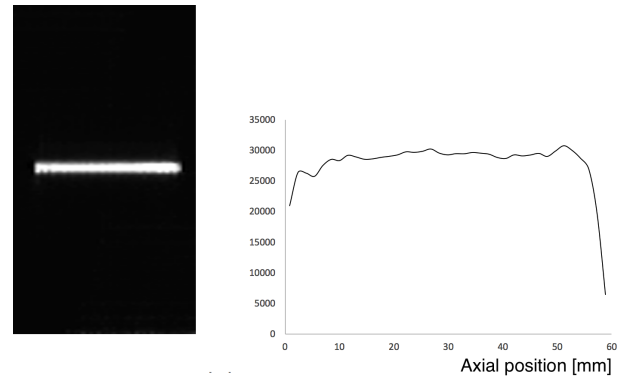

Fig. 14. Image of a line source aligned with the axis in the centre of the demonstrator (left) and the longitudinal profile (right).

\section{DISCUSSION}

We have generalised the previously developed iterative component-based ML algorithm for normalisation factor estimation by imposing the $3 \mathrm{D}$ symmetries for the full ring scanner. The algorithm is validated with $3 \mathrm{D}$ reconstruction of a simulated data with both full ring and partial ring scanner and with the experimental data with a partial ring demonstrator. It has the advantage that the efficiencies of the virtual crystals are automatically set to zero in the missing part of the detector ring in partial ring, no special treatment is required. The effect of the normalisation factors is evaluated by looking at the line profile of a selected slice of the obtained image before and after applying the normalisation factors. The uniformity of the image in both full ring and partial ring scanners is achieved in both transaxial and axial directions. There are some distortions in the reconstructed image in the partial ring scanner due to the incomplete angular coverage as expected which needs some 
other improvements in the reconstruction process, for instance using time-of-flight information, but this was out-of-scope for this paper. To validate the algorithm in $3 \mathrm{D}$, the product of the normalisation components for the full ring and partial ring are compared. This has been done by plotting the profile through a row of normalisation sinogram in a selected segment for both the scanners with the data obtained in simulation. Both the profiles give the same estimation for the product of the normalisation factors which proves that the $3 \mathrm{D}$ algorithm is valid and can be applied to the partial ring scanner. Note that we observed some differences in the estimated efficiencies and geometric factors, indicating there is some non-uniqueness in the components for the partial ring system, but not in the final product.

\section{ACKNOWLEDGMENT}

The author would like to thank Nikos Efthimiou for providing a software to convert the ROOT data to a format required by STIR.

\section{REFERENCES}

[1] T. Niknejad et al., Validation of a highly integrated SiPM readout system with a TOF-PET demonstrator, Journal of Instrumentation, 11 (12), 2016.

[2] D. Hogg et al., Maximum-likelihood estimation of normalisation factors for PET, IEEE Nuclear Science Symposium Conference Record, 4, 2001.

[3] M.W. Jacobson et al., Optimizability of loglikelihoods for the estimation of detector efficiencies and singles rates in PET, IEEE Nuclear Science Symposium Conference Record, 2008.

[4] K. Thielemans et al., STIR: software for tomographic image reconstruction release 2, Physics in Medicine and Biology, 57 (4), 2012.

[5] S. Jan et al., GATE: a simulation toolkit for PET and SPECT, Physics in Medicine and Biology, 49 (19), 2004.

[6] National Elecrical Manufacturers Association. Performance measurements for small animal Positron Emission Tomographs (PETs), Standards Publication NU 4-2008, Rosslyn, VA, 2008. 